\title{
ROBUST APERIODIC-DISTURBANCE REJECTION IN AN UNCERTAIN MODIFIED REPETITIVE-CONTROL SYSTEM
}

\author{
LAN ZHOU $^{a, *}$, JinhuA SHE ${ }^{b, c}, \mathrm{CHAOYI} \mathrm{LI}^{a}, \mathrm{CHANGZHONG} \mathrm{PAN}^{a}$ \\ ${ }^{a}$ School of Information and Electrical Engineering \\ Hunan University of Science and Technology, Xiangtan 411201, China \\ e-mail: zhoulan75@163.com \\ ${ }^{b}$ School of Automation \\ China University of Geosciences, Wuhan, Hubei 430074, China \\ ${ }^{c}$ School of Engineering \\ Tokyo University of Technology, Tokyo 192-0982, Japan
}

\begin{abstract}
This paper concerns the problem of designing an EID-based robust output-feedback modified repetitive-control system (ROFMRCS) that provides satisfactory aperiodic-disturbance rejection performance for a class of plants with time-varying structured uncertainties. An equivalent-input-disturbance (EID) estimator is added to the ROFMRCS that estimates the influences of all types of disturbances and compensates them. A continuous-discrete two-dimensional model is built to describe the EID-based ROFMRCS that accurately presents the features of repetitive control, thereby enabling the control and learning actions to be preferentially adjusted. A robust stability condition for the closed-loop system is given in terms of a linear matrix inequality. It yields the parameters of the repetitive controller, the output-feedback controller, and the EID-estimator. Finally, a numerical example demonstrates the validity of the method.
\end{abstract}

Keywords: repetitive control, equivalent input disturbance, two-dimensional system, singular-value decomposition, linear matrix inequality.

\section{Introduction}

Repetitive control (RC) has a human-like learning capability. A repetitive controller performs learning through a delay positive-feedback loop, which is an internal model of a periodic signal. For a given periodic reference input, the repetitive controller adds the tracking error of the previous period to the present error to produce a control signal and gradually reduces the tracking error through repeated learning actions (Inoue et al., 1981).

The relative degree of the plant must be zero for a basic RC system (RCS) to be exponentially stable. To guarantee the stability of a strictly proper plant, which is the type that most control engineering applications deal with, a low-pass filter has to be inserted into the delay line. The resulting system is called a modified RCS (MRCS, Fig. 1) (Hara et al., 1988). In an MRCS, the low-pass filter relaxes the stability condition, but degrades

\footnotetext{
*Corresponding author
}

the tracking precision for signals in the high-frequency band. That is, there exists a trade-off between stability and tracking performance in an MRCS. Many strategies have been proposed to try to resolve the trade-off (Pipeleers et al., 2009; Hu et al., 2011; Chung and Chen, 2012). As an effective solution, Zhou et al. (2012; 2013; 2014b) and She et al. (2012) exploited the periodicity and continuity of RC and developed a two-dimensional (2D) model-based method. It enables the preferential adjustment of control and learning actions, and the designed systems exhibit both satisfactory robustness and good control performance.

One problem with $\mathrm{RC}$ is that it cannot reject, and may even amplify, the aperiodic disturbances since the repetition period of the repetitive controller is set to the period of the reference input. In control engineering practice, external disturbances are often unknown and complex, including components with different frequencies. Over the past few decades, a 


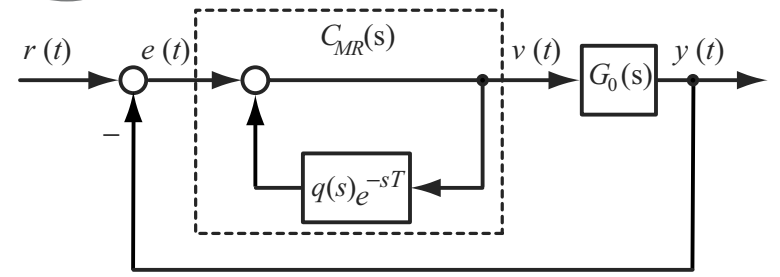

Fig. 1. Configuration of an MRCS.

considerable number of studies have been devoted to the estimation and rejection of an unknown disturbance. A disturbance observer (DOB) is commonly used for disturbance rejection (Chen et al., 2000; Miyazaki et al., 2006). They often implicitly assume that an equivalent input disturbance (EID) exists on the control input channel, but the design of the low-pass filter is frequently complicated because it has to guarantee both the causality of the DOB and the stability of the whole system.

A new method of EID estimation based on the control input and the output of the plant was devised by She et al. $(2008 ; 2011)$ that overcomes the drawbacks of the DOB-based methods. Wu et al. (2014), Zhou et al. (2014a) and Liu et al. (2014a) incorporated the EID method into an MRCS to improve the disturbance-rejection performance. For a plant without uncertainty, they separately designed the outer feedback loop and the inner disturbance attenuation one. However, there is a coupling relationship between the feedback controller and the EID estimator in the presence of time-varying uncertainties, so the outer and inner loops cannot be designed independently. Meanwhile, it shall also be noticed that the full state of the dynamic system is required in the approach (Zhou et al., 2014a; Liu et al., 2014a). To enable the EID-based RC method to handle a larger class of servo systems, this paper extends the state-feedback to output-feedback and presents the configuration of a robust EID-based MRCS.

This paper focuses especially on the problem of designing a robust output-feedback MRCS (ROFMRCS) with both periodic and aperiodic disturbances and with time-varying, structured uncertainties. First, we construct an EID-based ROFMRCS, in which an EID-estimator is used to estimate and compensate the influences of the total disturbance, including the uncertainties and all types of disturbances. Next, we build a continuous-discrete 2D model to describe the system. Then, a sufficient robust-stability condition in the form of a linear matrix inequality (LMI) is derived. Finally, simulations illustrate the tracking performance for a periodic reference input as well as the disturbance-rejection performance for both periodic and aperiodic disturbances.

Throughout this paper, $\mathbb{R}^{+}$is the set of nonnegative real numbers, $\mathbb{C}^{p}$ is the $p$-dimensional vector space over complex numbers, $\mathbb{Z}^{+}$is the set of nonnegative integers, $\aleph$ is the linear space of all the functions from $[0, T]$ to $\mathbb{C}^{p} . L_{2}\left(\mathbb{R}^{+}, \mathbb{C}^{p}\right)$ is the linear space of square integrable functions from $\mathbb{R}^{+}$to $\mathbb{C}^{p}$ and $\ell_{2}\left(\mathbb{Z}^{+}, \aleph\right)$ is the linear space of all the functions from $\mathbb{Z}^{+}$to $\aleph$, while

$$
\left[\begin{array}{ll}
\Xi & \Upsilon \\
* & \Omega
\end{array}\right]:=\left[\begin{array}{cc}
\Xi & \Upsilon \\
\Upsilon^{T} & \Omega
\end{array}\right] .
$$

\section{Problem description}

Consider a linear single-input, single-output (SISO) plant with time-varying structured uncertainties. Its state-space equation is

$$
\left\{\begin{aligned}
\dot{x}_{p}(t)= & {[A+\Delta A(t)] x_{p}(t) } \\
& +[B+\Delta B(t)] u(t)+B_{d} d(t), \\
y_{p}(t)= & C x_{p}(t)
\end{aligned}\right.
$$

where $x_{p}(t) \in \mathbb{R}^{n}$ is the state of the plant, $u(t), y_{p}(t) \in$ $\mathbb{R}$ are the control input and output variables, respectively, $d(t) \in \mathbb{R}^{n_{d}}$ is the disturbance input, while $A, B, B_{d}$, and $C$ are real constant matrices. Let the uncertainties of the plant be of the form

$$
\left[\begin{array}{cc}
\Delta A(t) & \Delta B(t)
\end{array}\right]=M E(t)\left[\begin{array}{ll}
N_{0} & N_{1}
\end{array}\right],
$$

where $M, N_{0}$, and $N_{1}$ are known constant matrices, while $E(t) \in \mathbb{R}^{n \times n}$ is a real, unknown, and time-varying matrix with Lebesgue measurable elements

$$
E^{T}(t) E(t) \leq I, \quad \forall t>0 .
$$

Two assumptions are made for the plant.

Assumption 1. $(A, B, C)$ is controllable and observable.

Assumption 2. ( $A, B, C$ ) has no zeros on the imaginary axis.

In the MRCS in Fig. 1] the repetition period of the repetitive controller, $T$, is the period of the reference input, $r(t)$. Here $e(t)[=r(t)-y(t)]$ is the tracking error between the reference input and the output. As explained by Hara et al. (1988), if the original closed-loop system without the delay line is stable and if

$$
\left\|q(s)\left(1+G_{0}(s)\right)^{-1}\right\|_{\infty}<1
$$

then the MRCS with minimal realization is exponentially stable.

A first-order low-pass filter

$$
q(s)=\frac{\omega_{c}}{s+\omega_{c}}
$$

is chosen to relax the stability condition of the MRCS and makes the system easy to design, where $\omega_{c}$ is the cutoff 


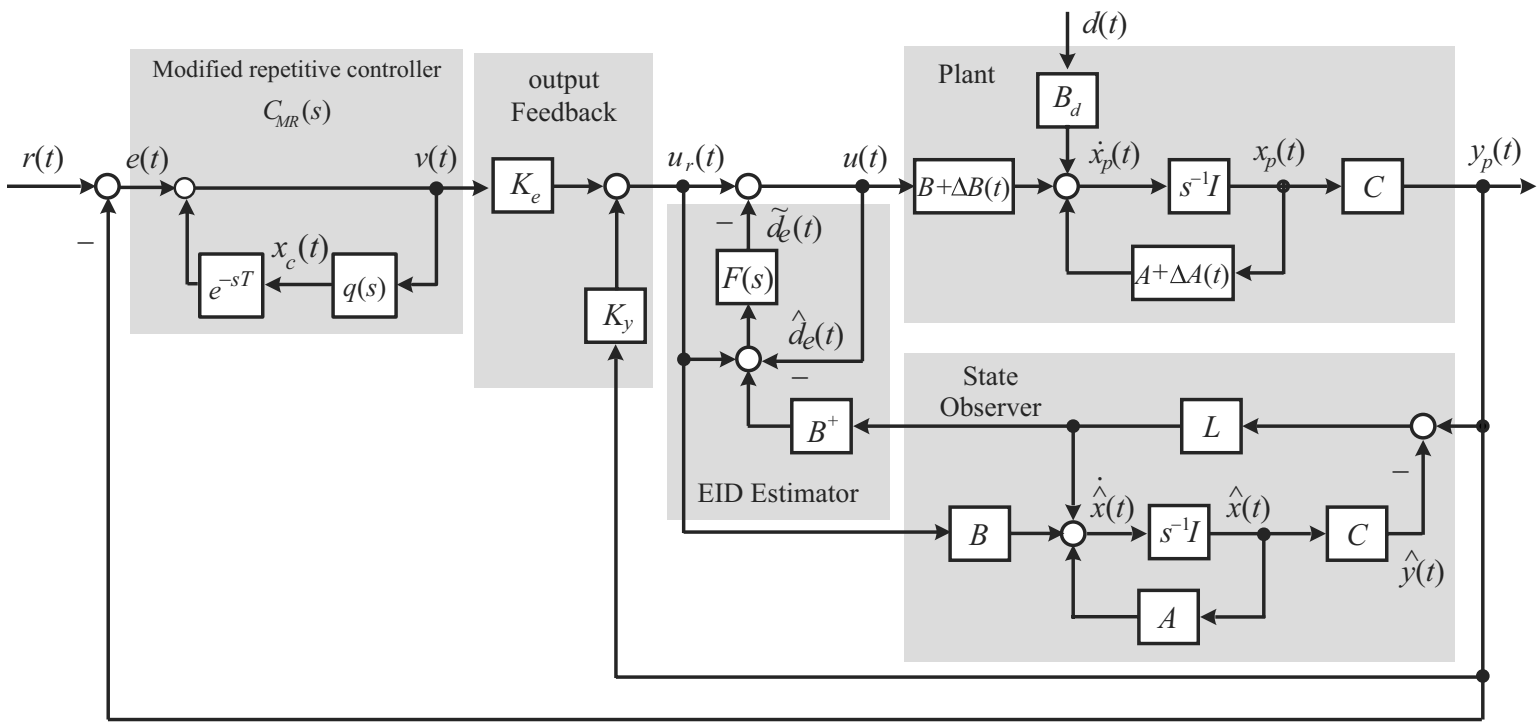

Fig. 2. Configuration of an EID-based ROFMRCS.

angular frequency. In (5), $\omega_{c}$ satisfies both the condition (4) and

$$
|q(j \omega)| \approx 1, \quad \omega \leq \omega_{q r}
$$

where $\omega_{q r}$ is the highest angular frequency of the reference input signals for the tracking (Hara et al., 1988). Thus, the state-space representation of the modified repetitive controller is

$$
\left\{\begin{array}{l}
\dot{x}_{c}(t)=-\omega_{c} x_{c}(t)+\omega_{c} x_{c}(t-T)+\omega_{c} e(t), \\
v(t)=e(t)+x_{c}(t-T)
\end{array}\right.
$$

where $x_{c}(t)$ and $v(t)$ are the state variable and the output variable of the repetitive controller, respectively.

The internal-mode principle tells us that the MRCS in Fig. 1 can reject both the periodic disturbance and the periodic uncertainties as long as their periods are the same as the repetition period, $T$. But for the aperiodic disturbances and the uncertainties with other frequencies, the repetitive controller cannot reject them, and may even amplify them. In this paper, the uncertainties and all types of disturbances are summarized as a total disturbance. To improve the disturbance-rejection performance, an EID estimator is inserted into the output-feedback MRCS to estimate the effects on the output of the generalized disturbance. This gives an EID-based ROFMRCS (Fig. 2).

A linear repetitive control law based on the outputs of the plant (1) and the repetitive controller (7) is

$$
u_{r}(t)=K_{e} v(t)+K_{y} y_{p}(t)
$$

where $K_{e} \in \mathbb{R}$ is the feedback gain of the repetitive controller and $K_{y} \in \mathbb{R}$ is the output-feedback gain.
Regarding the uncertainties of the plant as a kind of load disturbance, the plant (1) is written as

$$
\left\{\begin{aligned}
\dot{x}_{p}(t) & =A x_{p}(t)+B u(t) \\
& +\left[\Delta A(t) x_{p}(t)+\Delta B(t) u(t)+B_{d} d(t)\right], \\
y_{p}(t)= & C x_{p}(t) .
\end{aligned}\right.
$$

Employing the concept of EID and its existence in the work of She et al. (2008), there always exists an EID, $d_{e}(t)$, on the control input channel of the plant (9) that produces the same effect on the output as the total disturbance, $\Delta A(t) x(t)+\Delta B(t) u(t)+B_{d} d(t)$, does. This allows us to use the state equation

$$
\left\{\begin{array}{l}
\dot{x}(t)=A x(t)+B\left[u(t)+d_{e}(t)\right], \\
y(t)=C x(t)
\end{array}\right.
$$

to describe the plant 9 .

The basic idea of the EID approach is to design a disturbance estimator that estimates the EID, $d_{e}(t)$, and to use it to compensate for the total disturbance. Thus, incorporating the EID estimate into the RC law (8) yields the improved control law (Fig. 2)

$$
u(t)=u_{r}(t)-\tilde{d}_{e}(t),
$$

where $\tilde{d}_{e}(t)$ is an estimate of $d_{e}(t)$.

The following state observer is used to reproduce the state of the plant:

$$
\left\{\begin{array}{l}
\dot{\hat{x}}(t)=A \hat{x}(t)+B u_{r}(t)+L[y(t)-\hat{y}(t)], \\
\hat{y}(t)=C \hat{x}(t),
\end{array}\right.
$$

where $L$ is the observer gain, $\hat{x}(t) \in \mathbb{R}^{n}$ is the state variable, and $\hat{y}(t) \in \mathbb{R}$ is the output of the observer. 
As explained by She et al. (2008), an optimal estimate of the EID, $\hat{d}_{e}(t)$, is given by

$$
\hat{d}_{e}(t)=B^{+} L C[x(t)-\hat{x}(t)]+u_{r}(t)-u(t),
$$

where $B^{+}=\left(B^{T} B\right)^{-1} B^{T}$.

Taking into account that the output, $y(t)$, may contain measurement noise, we use a low-pass filter, $F(s)$, to filter the noise out of the estimate, $\hat{d}_{e}(t)$, and obtain $\tilde{d}_{e}(t)$. That is,

$$
\tilde{D}_{e}(s)=F(s) \hat{D}_{e}(s)
$$

where $\tilde{D}_{e}(s)$ and $\hat{D}_{e}(s)$ are the Laplace transforms of $\tilde{d}_{e}(t)$ and $\hat{d}_{e}(t)$.

As in the works of She et al. (2008) and Wu et al. (2014), in this paper we require that the disturbance estimates pass through the filter band

$$
\Omega_{r}:=\left\{\omega, 0 \leq \omega \leq \omega_{a r}\right\}
$$

where $\omega_{a r}$ is the highest angular frequency for disturbance rejection, and that they be not concerned about the rolloff speed of the gain of $F(j \omega)$ outside that band. That is, $F(s)$ satisfies

$$
|F(j \omega)| \approx 1, \quad \omega \leq \omega_{a r}
$$

To achieve this, a first-order low-pass filter is the best. Based on this, we choose

$$
F(s)=\frac{\alpha \omega_{f}}{s+\omega_{f}},
$$

where $\alpha \approx 1$ is a constant and $\omega_{f}$ is the cutoff angular frequency of the filter, which is usually set to a value more than five times greater than $\omega_{a r}$ (She et al., 2008; Wu et al., 2014). From (14) and (16), we have the state-space form of $F(s)$,

$$
\left\{\begin{array}{l}
\dot{x}_{f}(t)=A_{f} x_{f}(t)+B_{f} \hat{d}_{e}(t), \\
\tilde{d}_{e}(t)=C_{f} x_{f}(t)
\end{array}\right.
$$

where $x_{f}(t)$ is the state of the filter.

Remark 1. The EID-based ROFMRCS (Fig. 2) can be viewed as a conventional ROFMRCS combined with a state observer and an EID estimator. In the EID-based ROFMRCS, the repetitive controller and the output-feedback controller guarantee the tracking performance specifications and stability, while the incorporation of an EID estimate into the control input makes it capable of eliminating the total disturbance before it causes negative effect to the controlled plant. This is the main advantage over other RC methods.

\section{Design of the EID-based ROFMRCS}

In this section, we derive a sufficient robust stability condition for the closed-loop system in Fig. 2 and present a method of designing the controller parameters.

Since the stability of the system does not depend on exogenous signals, we first let both the reference input and the disturbance be zero:

$$
r(t)=0, \quad d(t)=0 .
$$

Let

$$
x_{\delta}(t)=x(t)-\hat{x}(t)
$$

be the error between the state of the actual plant and that of the observer. Then, the state equations of the plant (1) and the state observer (12) are respectively written as

$$
\begin{aligned}
& \dot{x}(t)=[A+\Delta A(t)] x(t)+[B+\Delta B(t)] u(t), \\
& \dot{\hat{x}}(t)=A \hat{x}(t)+L C x_{\delta}(t)+B u_{r}(t),
\end{aligned}
$$

and from 11, 17), 20), and 21) we have

$$
\begin{array}{r}
\dot{x}_{\delta}(t)=\Delta A(t) \hat{x}(t)+[A+\Delta A(t)-L C] x_{\delta}(t) \\
-[B+\Delta B(t)] C_{f} x_{f}(t)+\Delta B(t) u_{r}(t) .
\end{array}
$$

Also, the state equations (7) and (17) can be respectively rewritten as

$$
\begin{aligned}
\dot{x}_{c}(t)= & -\omega_{c} C \hat{x}(t)-\omega_{c} C x_{\delta}(t)-\omega_{c} x_{c}(t) \\
& +\omega_{c} x_{c}(t-T), \\
\dot{x}_{f}(t)= & B_{f} B^{+} L C x_{\delta}(t)+\left(A_{f}+B_{f} C_{f}\right) x_{f}(t) .
\end{aligned}
$$

Accordingly, the RC control law (8) becomes

$$
\begin{aligned}
u_{r}(t)= & \left(K_{y}-K_{e}\right) C \hat{x}(t) \\
& +\left(K_{y}-K_{e}\right) C x_{\delta}(t)+K_{e} x_{c}(t-T) .
\end{aligned}
$$

Employing the lifting technique (Yamamoto, 1994; Zhou et al., 2012), we convert a vector-valued continuous-time signal, $\xi(t)$, in the EID-based ROFMRCS (Fig. 2), into a function-valued discrete-time sequence, $\xi_{k}(\tau)$. Its element is denoted as $\xi(k, \tau)$ in this paper. That is,

$$
\begin{aligned}
& \xi(k, \tau)=\xi_{k}(\tau):=\mathcal{L}_{\mathcal{C}}[\xi(t)], \\
& t=k T+\tau, \quad \tau \in[0, T], \quad k \in \mathbb{Z}^{+},
\end{aligned}
$$

where $\mathcal{L}_{\mathcal{C}}$ is an isometric and isomorphic transformation between $L_{2}\left(\mathbb{R}_{+}, \mathbb{C}^{p}\right)$ and $\ell_{2}\left(\mathbb{Z}_{+}, \aleph\right)$. It gives us the following $2 \mathrm{D}$ representation:

$$
\begin{aligned}
& \left\{\begin{aligned}
\dot{\eta}(k, \tau)= & \bar{A} \eta(k, \tau)+\bar{A}_{1} \eta(k-1, \tau) \\
& +\bar{B} u_{r}(k, \tau), \\
e(k, \tau)= & -\bar{C} \eta(k, \tau),
\end{aligned}\right. \\
& u_{r}(k, \tau)=F_{p} \bar{C} \eta(k, \tau)+F_{e} \bar{C}_{1} \eta(k-1, \tau),
\end{aligned}
$$


where

$$
\begin{aligned}
& \eta(k, \tau)=\left[\hat{x}^{T}(k, \tau) \quad x_{\delta}^{T}(k, \tau)\right. \\
& \left.x_{f}^{T}(k, \tau) x_{c}^{T}(k, \tau)\right]^{T}, \\
& \bar{A}=\left[\begin{array}{lc}
A & L C \\
\Delta A(k, \tau) & A+\Delta A(k, \tau)-L C \\
0 & B_{f} B^{+} L C \\
-\omega_{c} C & -\omega_{c} C
\end{array}\right. \\
& \left.\begin{array}{cc}
0 & 0 \\
-(B+\Delta B(k, \tau)) C_{f} & 0 \\
A_{f}+B_{f} C_{f} & 0 \\
0 & -\omega_{c}
\end{array}\right], \\
& \bar{A}_{1}=\operatorname{diag}\left\{0,0,0, \omega_{\mathrm{c}}\right\}, \\
& \bar{B}=\left[\begin{array}{llll}
B^{T} & \Delta B^{T}(k, \tau) & 0 & 0
\end{array}\right]^{T} \text {, } \\
& \bar{C}=\left[\begin{array}{llll}
C & C & 0 & 0
\end{array}\right], \\
& \bar{C}_{1}=\left[\begin{array}{llll}
0 & 0 & 0 & 1
\end{array}\right] \text {, }
\end{aligned}
$$

and

$$
F_{p}=K_{y}-K_{e}, \quad F_{e}=K_{e}
$$

An RC process is basically continuous. In consequence, any state, $\xi(k, \tau)$, in the $2 \mathrm{D}$ model of the EID-based ROFMRCS in Fig. 2 satisfies the boundary conditions

$$
\begin{cases}\xi(-1, \tau)=0, & \tau \in[0, T), \\ \xi(k, 0)=\xi(k-1, T), & \tau \in\{1,2,3, \ldots\} .\end{cases}
$$

Meanwhile, from 29, the control gains in Fig. 2 $\mathrm{can}$ be rewritten as

$$
K_{e}=F_{e}, \quad K_{y}=F_{p}+F_{e} .
$$

Remark 2. Since we can view the past state as a kind of experience, the words control and learning mean that we use information about the present and previous periods, respectively, to produce the present control input (She et al., 2012). The 2D control law (28) contains the direct sum of the effects of control and learning. This allows the preferential adjustment of control and learning through the regulation of the feedback gains, $F_{p}$ and $F_{e}$. Note that since the low-pass filter (7) in an MRCS mixes the control and learning actions, we cannot adjust control and learning actions independently.

Substituting the control input 28 into the system (27) yields a representation of the closed-loop system in Fig. 2.

$$
\begin{aligned}
\dot{\eta}(k, \tau)= & A_{l} \eta(k, \tau)+A_{1 l} \eta(k-1, \tau) \\
& +M_{l}\left[\Gamma(k, \tau)+\Gamma_{1}(k, \tau)\right],
\end{aligned}
$$

where

$$
\begin{aligned}
A_{l}= & {\left[\begin{array}{cc}
A+B F_{p} C & L C+B F_{p} C \\
0 & A-L C \\
0 & B_{f} B^{+} L C \\
-\omega_{c} C & -\omega_{c} C \\
0 & 0 \\
-B C_{f} & 0 \\
A_{f}+B_{f} C_{f} & 0 \\
0 & -\omega_{c}
\end{array}\right], } \\
A_{1 l}= & {\left[\begin{array}{cccc}
0 & 0 & 0 & B F_{e} \\
0 & 0 & 0 & 0 \\
0 & 0 & 0 & 0 \\
0 & 0 & 0 & \omega_{c}
\end{array}\right], \quad M_{l}=\left[\begin{array}{c}
0 \\
M \\
0 \\
0
\end{array}\right], }
\end{aligned}
$$

$$
\Gamma(k, \tau)=F(k, \tau) \Psi \eta(k, \tau),
$$$$
\Gamma_{1}(k, \tau)=F(k, \tau) \Psi_{1} \eta(k-1, \tau),
$$$$
\Psi=\left[\begin{array}{lll}
N_{0}+N_{1} F_{p} C & N_{0}+N_{1} F_{p} C-N_{1} C_{f} & 0
\end{array}\right],
$$$$
\Psi_{1}=\left[\begin{array}{cccc}
0 & 0 & 0 & N_{1} F_{e}
\end{array}\right] \text {. }
$$

From (3) we have

$$
\begin{aligned}
\Gamma^{T}(k, \tau) \Gamma(k, \tau) & \leq \eta^{T}(k, \tau) \Psi^{T} \Psi \eta(k, \tau), \\
\Gamma_{1}^{T}(k, \tau) \Gamma_{1}(k, \tau) & \leq \eta^{T}(k-1, \tau) \Psi_{1}^{T} \Psi_{1} \eta(k-1, \tau) .
\end{aligned}
$$

Assume that the singular-value decomposition (SVD) (Zhou et al., 1996) of the output matrix $C$ is

$$
C=U\left[\begin{array}{ll}
S & 0
\end{array}\right] V^{T},
$$

where $S$ is a diagonal matrix with positive, diagonal elements in decreasing order, 0 is a zero matrix, and $U$ and $V$ are unitary matrices.

Lemma 1. (Ho and Lu, 2003) For the SVD (35), if $X \in$ $\mathbb{R}^{n \times n}$ is a symmetric matrix, then there exists a matrix, $\bar{X} \in \mathbb{R}^{m \times m}$, such that $C X=\bar{X} C$ holds if and only if

$$
X=V \operatorname{diag}\left\{X_{11}, X_{22}\right\} V^{T},
$$

where $X_{11} \in \mathbb{R}^{m \times m}$ and $X_{22} \in \mathbb{R}^{(n-m) \times(n-m)}$.

Lemma 2. (Schur complement) (Khargonek et al., 1990) For any real matrix $\Sigma=\Sigma^{T}$, the following assertions are equivalent:

$$
\begin{aligned}
& \text { 1. } \Sigma=\left[\begin{array}{cc}
S_{11} & S_{12} \\
\star & S_{22}
\end{array}\right]<0, \\
& \text { 2. } S_{11}<0 \text { and } S_{22}-S_{12}^{T} S_{11}^{-1} S_{12}<0 \text {, } \\
& \text { 3. } S_{22}<0 \text { and } S_{11}-S_{12} S_{22}^{-1} S_{12}^{T}<0 \text {. }
\end{aligned}
$$

Note that the dynamic boundary conditions in 30 reveal that the EID-based ROFMRCS in Fig. 2 is stable if and only if there exists a semi-positive definite functional $V(k, \tau)$ decreasing monotonically in every interval $[k T,(k+1) T], k \in\{0,1,2, \ldots\}$. Accordingly, we have the following result. 
Theorem 1. For a given cutoff angular frequency, $\omega_{c}$, and two positive scalars, $\alpha$ and $\beta$, the system (32) is robustly stable if there exist symmetrical positive-definite matrices $X_{11}, X_{22}, \bar{X}_{11}, \bar{X}_{22}, X_{3}, X_{4}, Y_{1}, Y_{2}, Y_{3}$, and $Y_{4}$, and arbitrary matrices $W_{1}, W_{2}, W_{3}$, and $W_{4}$, such that the following LMI holds:

$$
\left[\begin{array}{cccc}
\Theta & \Lambda & \Xi & X \\
* & Y & \Pi & 0 \\
* & * & \Upsilon & 0 \\
* & * & * & Y
\end{array}\right]<0
$$

where

$$
\begin{aligned}
& \Theta=\left[\begin{array}{cccc}
\Theta_{11} & W_{4} C+B W_{2} C & 0 & -\alpha \omega_{c} X_{1} C^{T} \\
* & \Theta_{22} & \Theta_{23} & -\omega_{c} X_{2} C^{T} \\
* & * & \Theta_{33} & 0 \\
* & * & * & -2 \beta \omega_{c} X_{4}
\end{array}\right] \\
& \Lambda=\left[\begin{array}{cccc}
0 & 0 & 0 & \beta B W_{3} \\
0 & 0 & 0 & 0 \\
0 & 0 & 0 & 0 \\
0 & 0 & 0 & \beta \omega_{C} Y_{4}
\end{array}\right] \text {, } \\
& \Xi=\left[\begin{array}{cccc}
0 & 0 & \alpha X_{1} N_{0}^{T}+\alpha C^{T} W_{1}^{T} N_{1}^{T} & 0 \\
M & M & X_{2} N_{0}^{T}+C^{T} W_{2}^{T} N_{1}^{T} & 0 \\
0 & 0 & -X_{3} C_{f}^{T} N_{1}^{T} & 0 \\
0 & 0 & 0 & 0
\end{array}\right], \\
& X=\operatorname{diag}\left\{\alpha \mathrm{X}_{1}, \mathrm{X}_{2}, \mathrm{X}_{3}, \beta \mathrm{X}_{4}\right\} \text {, } \\
& Y=\operatorname{diag}\left\{-\mathrm{Y}_{1},-\mathrm{Y}_{2},-\mathrm{Y}_{3},-\beta \mathrm{Y}_{4}\right\} \text {, } \\
& \Pi=\operatorname{diag}\left\{0,0,0, \mathrm{~W}_{3}^{\mathrm{T}} \mathrm{N}_{1}^{\mathrm{T}}\right\} \text {, } \\
& \Upsilon=\operatorname{diag}\{-\mathrm{I},-\mathrm{I}, \quad-\mathrm{I},-\mathrm{I}\}, \\
& X_{1}=V \operatorname{diag}\left\{\mathrm{X}_{11}, \mathrm{X}_{22}\right\} \mathrm{V}^{\mathrm{T}}, \\
& X_{2}=V \operatorname{diag}\left\{\overline{\mathrm{X}}_{11}, \overline{\mathrm{X}}_{22}\right\} \mathrm{V}^{\mathrm{T}} \text {, } \\
& \Theta_{11}=\alpha A X_{1}+\alpha X_{1} A^{T}+\alpha B W_{1} C+\alpha C^{T} W_{1}^{T} B^{T}, \\
& \Theta_{22}=X_{2} A^{T}+A X_{2}-W_{4} C-C^{T} W_{4}^{T}, \\
& \Theta_{23}=-B C_{f} X_{3}+C^{T} W_{4}^{T} B^{+^{T}} B_{f}^{T} \text {, } \\
& \Theta_{33}=A_{f} X_{3}+X_{3} A_{f}^{T}+B_{f} C_{f} X_{3}+X_{3}^{T} C_{f}^{T} B_{f}^{T} \text {. }
\end{aligned}
$$

and

Furthermore, the 2D gains in (28) are

$$
F_{p}=W_{1} U S X_{11}^{-1} S^{-1} U^{T}, \quad F_{e}=W_{3} Y_{4}^{-1},
$$

and the observer gain is

$$
L=W_{4} U S \bar{X}_{11}^{-1} S^{-1} U^{T} .
$$

Proof. Let $P_{i}=X_{i}^{-1}, \quad Q_{i}=Y_{i}^{-1}, \quad i=1,2,3, \ldots$ Choose a Lyapunov functional candidate to be

$$
\begin{aligned}
V(k, \tau)= & \eta^{T}(k, \tau) P \eta(k, \tau) \\
& +\int_{\tau-T}^{\tau} \eta^{T}(k, s) Q \eta(k, s) \mathrm{d} s
\end{aligned}
$$

where

$$
\begin{aligned}
& P=\operatorname{diag}\left\{\frac{1}{\alpha} P_{1}, P_{2}, P_{3}, \frac{1}{\beta} P_{4}\right\}, \\
& Q=\operatorname{diag}\left\{Q_{1}, Q_{2}, Q_{3}, \frac{1}{\beta} Q_{4}\right\} .
\end{aligned}
$$

Along the time trajectory of $\underline{32}$

$$
\frac{\mathrm{d} V(k, \tau)}{\mathrm{d} \tau}=\varphi^{T}(k, \tau) \Sigma \varphi(k, \tau),
$$

where

$$
\begin{gathered}
\varphi^{T}(k, \tau)=\left[\eta^{T}(k, \tau)\right. \\
\Sigma=\left[\begin{array}{cccc}
A_{l}^{T} P+P A_{l}+Q & P A_{1 l} & P M_{l} & P M_{l} \\
* & -Q & 0 & 0 \\
* & * & 0 & 0 \\
* & * & * & 0
\end{array}\right] .
\end{gathered}
$$

Then, from (32) and (42), we have

$$
\begin{gathered}
\frac{\mathrm{d} V(k, \tau)}{\mathrm{d} \tau}-\left[\Gamma^{T}(k, \tau) \Gamma(k, \tau)-\eta^{T}(k, \tau) \Psi^{T} \Psi \eta(k, \tau)\right. \\
\left.+\Gamma_{1}^{T}(k, \tau) \Gamma_{1}(k, \tau)-\eta^{T}(k-1, \tau) \Psi_{1}^{T} \Psi_{1} \eta(k-1, \tau)\right] \\
=\varphi^{T}(k, \tau) \tilde{\Sigma} \varphi(k, \tau),
\end{gathered}
$$

where

$$
\begin{aligned}
\tilde{\Sigma} & =\Sigma-\tilde{I}+\tilde{\Psi}^{T} \tilde{\Psi}+\tilde{\Psi}_{1}^{T} \tilde{\Psi}_{1}, \\
\tilde{I} & =\operatorname{diag}\{0,0,0,0,0,0,0,0, \mathrm{I}, \mathrm{I}\}, \\
\tilde{\Psi} & =\left[\begin{array}{lllllll}
\Psi & 0 & 0 & 0 & 0 & 0 & 0
\end{array}\right], \\
\tilde{\Psi}_{1} & =\left[\begin{array}{lllllll}
\Psi_{1} & 0 & 0 & 0 & 0 & 0 & 0
\end{array}\right] .
\end{aligned}
$$

From (33), 34), and 433, $\tilde{\Sigma}<0$ implies that, for any $\varphi(k, \tau) \neq 0, \mathrm{~d} V(t) / \mathrm{d} t<0$. Also, using Lemma2, $\tilde{\Sigma}<0$ is equivalent to the inequality

$$
\Omega=\left[\begin{array}{ccccccc}
\Omega_{11} & \tilde{\Psi} & \tilde{\Psi}_{1} & \tilde{Q}_{1} & \tilde{Q}_{2} & \tilde{Q}_{3} & \tilde{Q}_{4} \\
* & -I & 0 & 0 & 0 & 0 & 0 \\
* & * & -I & 0 & 0 & 0 & 0 \\
* & * & * & -Q_{1} & 0 & 0 & 0 \\
* & * & * & * & -Q_{2} & 0 & 0 \\
* & * & * & * & * & -Q_{3} & 0 \\
* & * & * & * & * & * & -Q_{4}
\end{array}\right]<0
$$

where

$$
\begin{aligned}
\Omega_{11} & =\Sigma-\tilde{I}-\tilde{Q}, \\
\tilde{Q} & =\operatorname{diag}\left\{\mathrm{Q}_{1}, \mathrm{Q}_{2}, \mathrm{Q}_{3}, \mathrm{Q}_{4}, 0,0,0,0,0,0\right\}, \\
\tilde{Q}_{1} & =\left[\begin{array}{llllllllll}
Q_{1}^{T} & 0 & 0 & 0 & 0 & 0 & 0 & 0 & 0 & 0
\end{array}\right]^{T}, \\
\tilde{Q}_{2} & =\left[\begin{array}{llllllllll}
0 & Q_{2}^{T} & 0 & 0 & 0 & 0 & 0 & 0 & 0 & 0
\end{array}\right]^{T}, \\
\tilde{Q}_{3} & =\left[\begin{array}{llllllllll}
0 & 0 & Q_{3}^{T} & 0 & 0 & 0 & 0 & 0 & 0 & 0
\end{array}\right]^{T}, \\
\tilde{Q}_{4} & =\left[\begin{array}{llllllllll}
0 & 0 & 0 & Q_{4}^{T} & 0 & 0 & 0 & 0 & 0 & 0
\end{array}\right]^{T} .
\end{aligned}
$$


Also, from (35), 37), (38), and Lemma 1, there exist

$$
\bar{X}_{1}=U S X_{11} S^{-1} U^{T}, \quad \bar{X}_{2}=U S \bar{X}_{11} S^{-1} U^{T}
$$

such that

$$
C X_{1}=\bar{X}_{1} C, \quad C X_{2}=\bar{X}_{2} C .
$$

Define

$$
\left\{\begin{array}{l}
W_{1}=F_{p} \bar{X}_{1}, W_{2}=F_{p} \bar{X}_{2}, \\
W_{3}=F_{e} Y_{4}, W_{4}=L \bar{X}_{2} .
\end{array}\right.
$$

Pre- and post-multiplying the matrix on the left side of (44) by $\operatorname{diag}\left\{P^{-1}, Q^{-1}, I, I, I, I, Q^{-1}\right\}$ yields the required LMI (36).

Finally, we obtain the controller gains 39 and 40 from (47).

Remark 3. Theorem 1 presents an LMI-based sufficient condition for the robust stability of the closed-loop ROFMRCS 32). The condition can be used directly to design the control gains in Fig. 2. Two tuning parameters, $\alpha$ and $\beta$, enable the preferential adjustment of control and learning actions. More specifically, $\alpha$ is used to adjust the weighting matrix $P_{1}$ and $\beta$ is used to adjust the weighting matrices $P_{4}$ and $Q_{4}$. Thus they change the feasible solutions, $F_{p}$ and $F_{e}$, in 39 . Moreover, the tuning parameters can reduce the conservativeness of the robust stability condition (36). For the adjustment of the control performance, compared with the results of Liu $e t$ al. (2014b), it is more effective to improve both transient and steady-state tracking performance through adjusting the gains in the 2D control law.

Note that Theorem 1 does not consider the information of the size of time delay, $T$, on the stability. Since the stability of the system is independent of the size of time delay, Theorem 1 is conservative to some extent. But considering that the period of a reference input changes for different tasks, it is much practical to design the EID-based ROFMRCS without using the information of the period. Moreover, it is also known that an MRCS is not very conservative when $T$ is not very small.

In this paper, we employ the performance index

$$
J_{n}=\frac{1}{2} \sum_{k=1}^{n} \int_{(k-1) T}^{k T} e^{2}(t) \mathrm{d} t
$$

to evaluate the system's overall performance and use it as a criterion for the selection of the tuning parameters, $\alpha$ and $\beta$.

In addition, from Theorem 1 the sufficient stability condition and the control parameters of the EID-based ROFMRCS in Fig. 2 for the nominal plant

$$
\left\{\begin{array}{l}
\dot{x}_{p}(t)=A x_{p}(t)+B u(t)+B_{d} d(t), \\
y_{p}(t)=C x_{p}(t),
\end{array}\right.
$$

are given by the following corollary.
Corollary 1. For a given cutoff angular frequency, $\omega_{c}$, and two positive scalars, $\alpha$ and $\beta$, the closed-loop system (32) for the nominal plant (49) is asymptotically stable if there exist symmetrical positive-definite matrices $X_{11}$, $X_{22}, \bar{X}_{11}, \bar{X}_{22}, X_{3}, X_{4}, Y_{1}, Y_{2}, Y_{3}$, and $Y_{4}$, and arbitrary matrices $W_{1}, W_{2}, W_{3}$, and $W_{4}$, such that the LMI

$$
\left[\begin{array}{ccc}
\Theta & \Lambda & X \\
* & Y & 0 \\
* & * & Y
\end{array}\right]<0
$$

holds, where $\Theta, \Lambda, X$, and $Y$ are defined in (36). Furthermore, the $2 D$ gains in (28) are

$$
F_{p}=W_{1} U S X_{11}^{-1} S^{-1} U^{T}, \quad F_{e}=W_{3} Y_{4}^{-1},
$$

and the observer gain is

$$
L=W_{4} U S \bar{X}_{11}^{-1} S^{-1} U^{T} .
$$

The result of Theorem 1 yields the following design algorithm for the EID-based ROFMRCS in Fig. 2.

Algorithm 1. Design of an EID-based ROFMRCS.

Step 1. Use the period of the periodic reference input to determine the delay constant, $T$, of the repetitive controller.

Step 2. Design a low-pass filter, $q(s)$, in (5) that satisfies the conditions (4) and (6)

Step 3. Select a low-pass filter, $F(s)$, in the form of (16), such that (15) holds.

Step 4. Find values of $\alpha$ and $\beta$ under the LMI-based condition (36) for which a $J_{n}$ is a minimum.

Step 5. Calculate $K_{e}$ and $K_{y}$ using Theorem 1 and Eqn. (31).

\section{Numerical example}

Assume that the parameters of the uncertain plant (1) are

$$
\left\{\begin{array}{l}
A=\left[\begin{array}{cc}
-1 & 3 \\
2 & -3
\end{array}\right], B=\left[\begin{array}{l}
1 \\
0
\end{array}\right], B_{d}=\left[\begin{array}{c}
1 \\
1.2
\end{array}\right], \\
C=\left[\begin{array}{ll}
1 & 0
\end{array}\right], M=\left[\begin{array}{ll}
1 & 0 \\
0 & 1
\end{array}\right], N_{0}=\left[\begin{array}{cc}
0.1 & 0 \\
0 & 0.01
\end{array}\right], \\
N_{1}=\left[\begin{array}{c}
0.01 \\
0
\end{array}\right], E(t)=\left[\begin{array}{cc}
\sin 0.6 \pi t & 0 \\
0 & \sin 0.6 \pi t
\end{array}\right] .
\end{array}\right.
$$

Consider the problem of tracking the reference input

$$
r(t)=\sin (2 \pi t)
$$

Thus, the repetition period is $T=1 \mathrm{~s}$.

The disturbance

$$
d(t)=k_{1} d_{1}(t)+k_{2} d_{2}(t)+k_{3} d_{3}(t)
$$




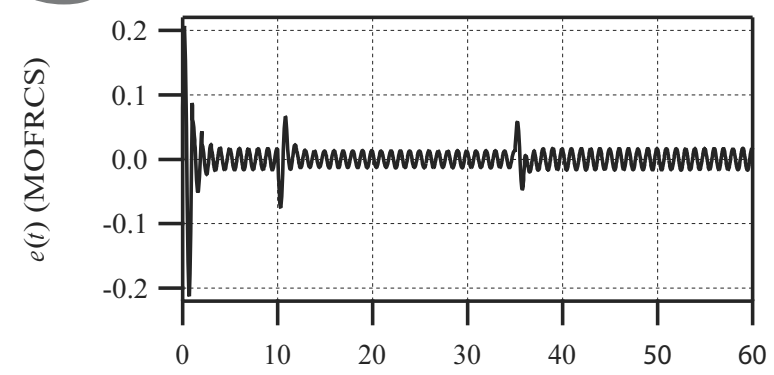

(a) ${ }^{t[\mathrm{~s}]}$

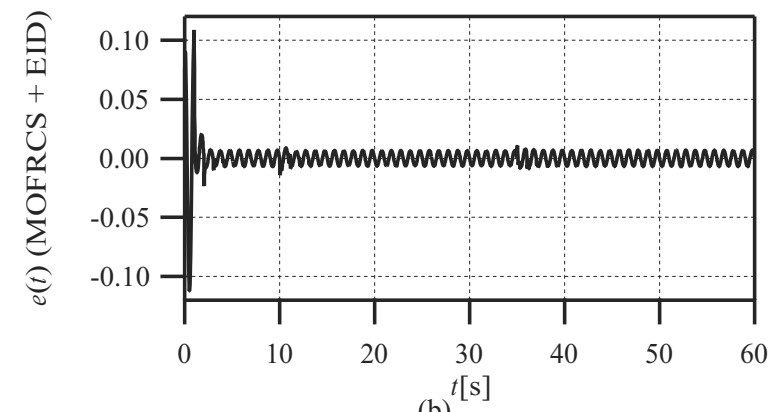

(b)

Fig. 3. Simulation results for $r(t)$ and the periodic disturbance $d_{1}(t)$ in 56 .

is added to the plant, where $k_{1}, k_{2}$, and $k_{3}$ are constants, and

$d_{1}(t)= \begin{cases}0, & t<10, \\ 0.7 \cos (2 \pi t)+3 \sin (2 \pi t), & 10 \leq t \leq 35, \\ 0, & t>35,\end{cases}$
$d_{2}(t)= \begin{cases}0, & t<10, \\ 2 \sin (\pi t)+0.2 \sin (3 \pi t), & 10 \leq t \leq 35, \\ 0, & t>35,\end{cases}$
$d_{3}(t)= \begin{cases}0, & t<0, \\ 2(\tanh (t-9)+\tan (t-10)), & 0 \leq t \leq 35, \\ 0, & t>35\end{cases}$

In 56, $d_{1}(t)$ is a periodic disturbance with the period of $T=1 \mathrm{~s}$, which is the same as that of the repetitive controller, $d_{2}(t)$ is also periodic, but its period is $6 \mathrm{~s}$, which is different from that of the repetitive controller, and $d_{3}(t)$ is aperiodic. Accordingly, the repetitive controller can reject $d_{1}(t)$, but it cannot reject $d_{2}(t)$ or $d_{3}(t)$.

An EID-based ROFMRCS was designed by following the design procedure in Section 3 using the Robust Control Toolbox of MATLAB R2015. First, the cutoff angular frequency of the low-pass filter $q(s)$ was chosen to be

$$
\omega_{c}=100 \mathrm{rad} / \mathrm{s} .
$$

Next, since the highest angular frequency of the disturbance, $d(t)$, is $3 \pi \mathrm{rad} / \mathrm{s}$, the upper bound on the frequency $\omega_{a r}$ is chosen to be $10 \mathrm{rad} / \mathrm{s}$. From [15, we

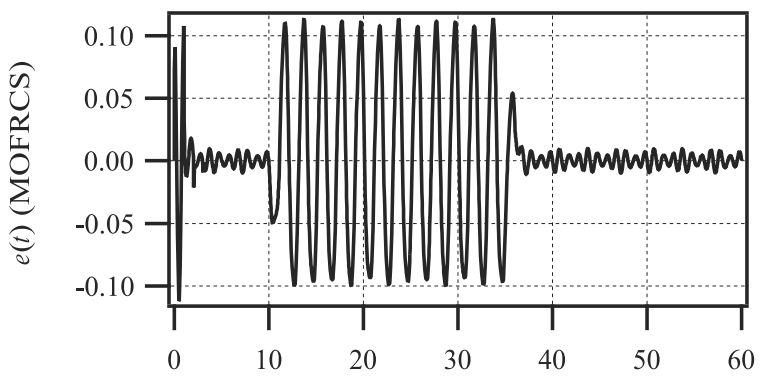

(a) $t[s$

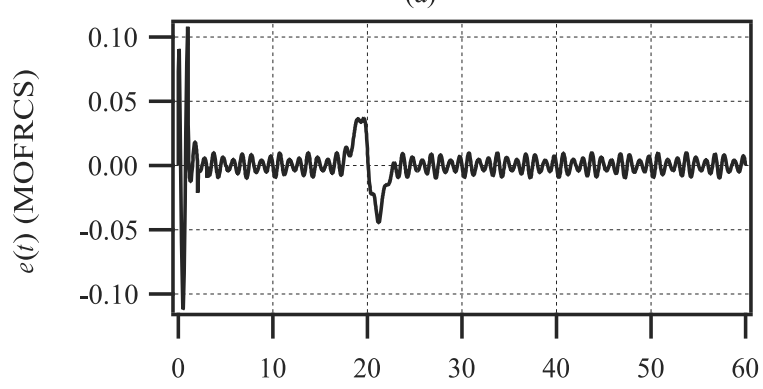

(b) $t[s]$

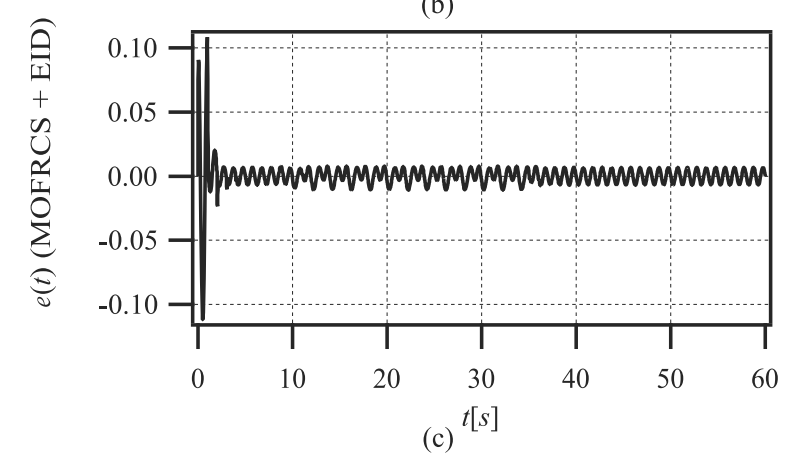

Fig. 4. Simulation results for $r(t)$ and the disturbance $d(t)$ in (55) for $k_{2}=1, k_{1}=k_{3}=0$ (a), $k_{3}=1, k_{1}=k_{2}=$ 0 (b), $k_{1}=0, k_{2}=k_{3}=1$ (c).

set

$$
F(s)=\frac{100}{s+101} .
$$

This choice guarantees not only that the gain of the filter is very close to one, but also that the phase lag is very small for $\omega \leq \omega_{a r}$. The parameters of the state-space form of the filter 17 are

$$
A_{f}=-101, \quad B_{f}=100, \quad C_{f}=1 .
$$

Then, according to Remark 3, two tuning parameters, $\alpha$ and $\beta$, in the stability condition (36) allow the preferential adjustment of control and learning. In the adjusting process, we employed the index $J_{60}$ in (48) to examine the system's overall performance. Applying the fixed-step method for $\alpha, \beta \in(0,1000]$ and the optimization algorithm

$$
\min J_{60} \text { so that LMI (36) holds }
$$



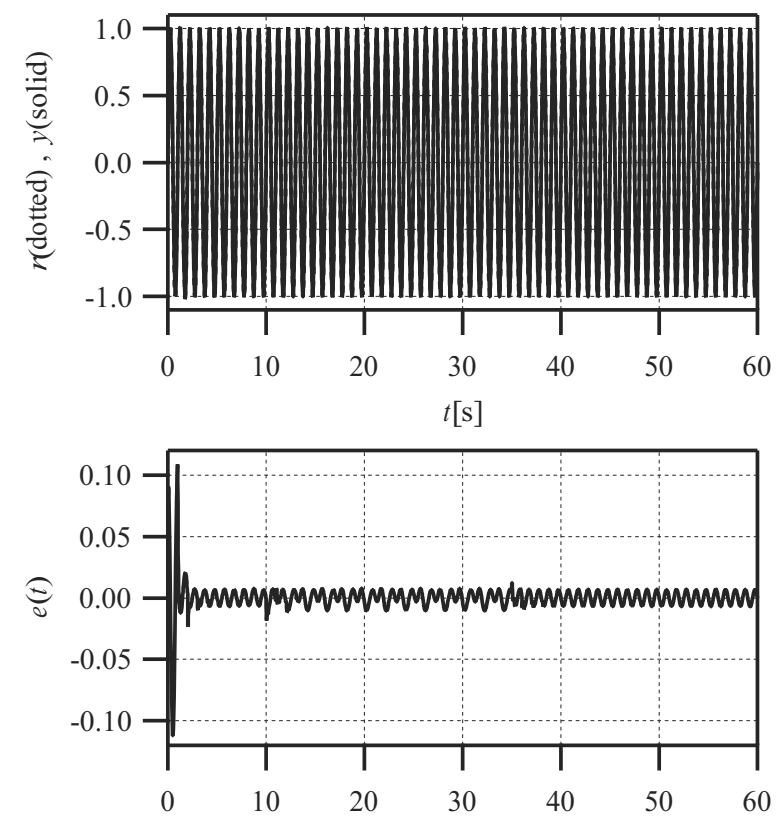

$t[\mathrm{~s}]$

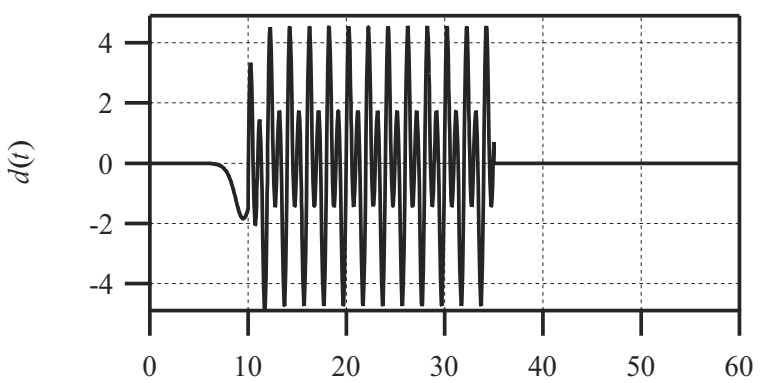

$t[\mathrm{~s}]$

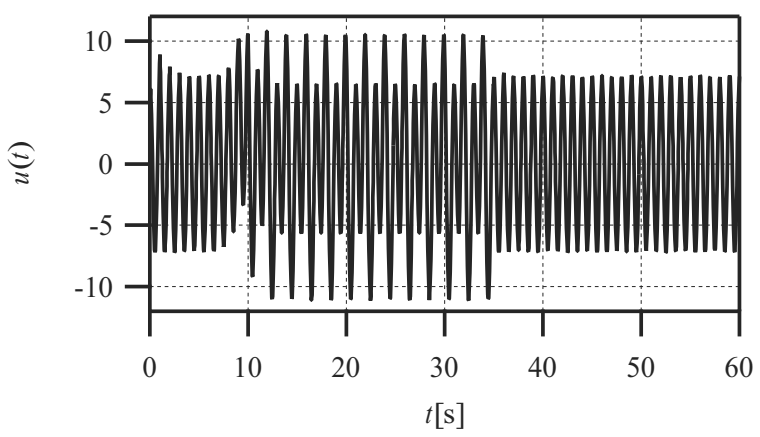

Fig. 5. Simulation results of the EID-based ROFMRCS for $r(t)$ and $d(t)$ in 55 for $k_{1}=k_{2}=k_{3}=1$.

yielded the best tuning parameters,

$$
\alpha=107.0, \quad \beta=4.0 \text {. }
$$

The corresponding control gains are

$$
\left\{\begin{array}{l}
K_{e}=64.4714, K_{y}=1.0022, \\
L=\left[\begin{array}{ll}
13.3968 & 4.9824
\end{array}\right]^{T},
\end{array}\right.
$$

and $J_{60}=0.0306$.

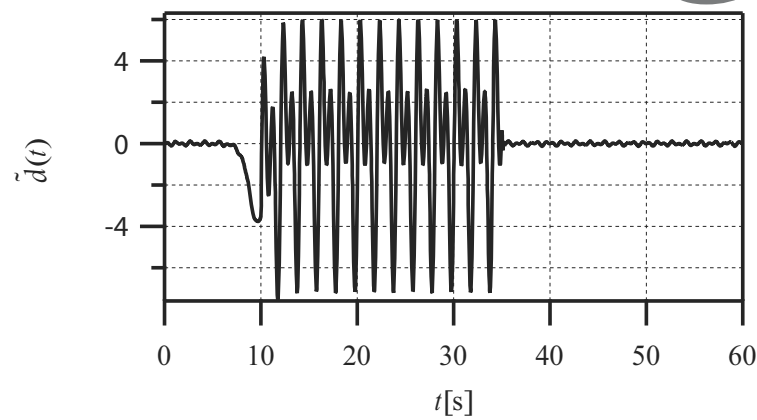

Fig. 6. EID estimate $\tilde{d}(t)$

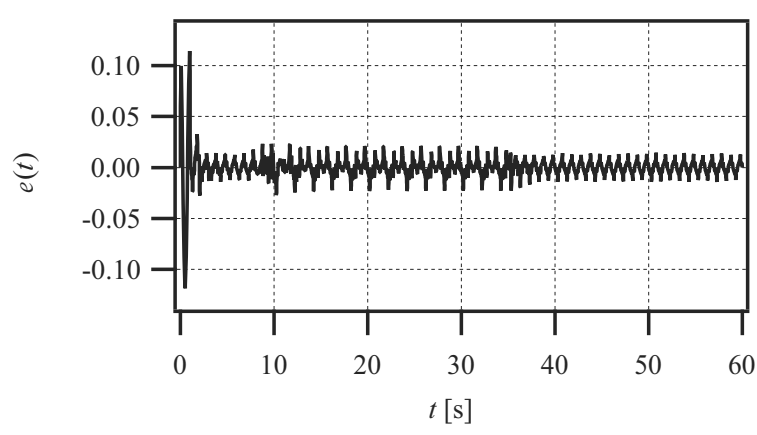

Fig. 7. Tracking error for the EID-based ROFMRCS with the dead zone $([-1,1])$ in the control input.

Simulations were carried out to compare the tracking performances for both periodic disturbances and aperiodic disturbances between the conventional ROFMRCS and the EID-based ROFMRCS.

Figure 3 shows the simulation results for the periodic reference input, $r(t)$, and the periodic disturbance, $d(t)=d_{1}(t)$. We find that (a) the conventional ROFMRCS rejects $d(t)$ in the steady state and (b) the EID-based ROFMRCS provides much better transient disturbance-rejecting performance than the conventional ROFMRCS does.

The simulation results in Fig. 4 show that the periodic disturbance $d_{2}(t)$ and the aperiodic disturbance $d_{3}(t)$ degrade the system tracking performance of the conventional ROFMRCS. Meanwhile, for $d(t)=d_{2}(t)+$ $d_{3}(t)$, the largest peak-to-peak tracking error of the EID-based ROFMRCS (Fig. 4 (c)) is 0.0175, and it is only $8.14 \%$ of that of the conventional ROFMRCS without EID compensation.

The simulation results for the EID-based ROFMRCS are shown in Fig. 5. The closed-loop system is asymptotically stable and it suppresses the transient tracking error caused by $d(t)=d_{1}(t)+d_{2}(t)+d_{3}(t)$ for $t \in[10 \mathrm{~s}, 35 \mathrm{~s}]$. From the EID estimate $\tilde{d}(t)$ (Fig. 6), we find that the EID estimator automatically produced a satisfactory compensation for the total disturbance, and thereby greatly improved the disturbance-rejecting performance. Compared with the work of She et al. 
(2010), in which parameter optimization is used to suppress the periodic uncertainties whose period is different from the repetition period of the repetitive controller, we can actively suppress the total disturbance by adding an EID-estimator into the MRCS, and need not regulate the parameters by trial and error.

In a practical application, the nonlinearity may degrade tracking performance. To examine its influence and verify the validity of our method, we carried out simulations for the case where there was a dead zone $\left(\left[\begin{array}{ll}-1 & 1\end{array}\right]\right)$ in the control input. The simulation result (Fig. 7) shows that the control system remained robustly stable and that the steady-state error caused by $r(t)$ and $d(t)=d_{1}(t)+d_{2}(t)+d_{3}(t)$ was suppressed to a low level even when there was a dead zone in the actuator. This implies that the improved RC control law also compensates effectively for the dead zone.

\section{Conclusion}

An aperiodic-disturbance rejection method for a strictly proper ROFMRCS with time-varying uncertainties was developed in this paper. An EID-based ROFMRCS was constructed, in which an EID estimator was added to estimate and compensate the total disturbance, including uncertainties and aperiodic disturbances with different frequencies. A robust stability condition was derived, and the control and learning actions involved in the $\mathrm{RC}$ process were adjusted preferentially by means of two tuning parameters. The advantage of this method is that we do not need any information about the disturbances, nor do we need to construct an inverse model to compensate for them. It handles the admissible uncertainties and adequately suppresses the aperiodic disturbances. Simulation results show that the EID-based ROFMRCS provides satisfactory tracking and disturbance rejection performance, even for a dead zone in the control input. In addition, the results are easy to extend to a multi-input, multi-output case.

\section{Acknowledgment}

This work was supported in part by the National Natural Science Foundation of China (nos. 61203010, 61473313, and 61403135).

\section{References}

Chen, X., Fukuda, T. and Young, K.D. (2000). A new nonlinear robust disturbance observer, Systems and Control Letters 41(3): 189-199.

Chung, C.H. and Chen, M.S. (2012). A robust adaptive feedforward control in repetitive control design for linear systems, Automatica 48(1): 183-190.

Hara, S., Yamamoto, Y. and Omata, T. (1988). Repetitive control system: A new type servo system for periodic exogenous signals, IEEE Transactions on Automatic Control 33(7): 659-668.

Ho, D.W.C. and Lu, G. (2003). Robust stabilization for a class of discrete-time nonlinear system via output feedback: The unified LMI approach, International Journal of Control 76(7): 105-115.

Hu, C.X., Yao, B., Chen, Z. and Wang, Q.F. (2011). Adaptive robust repetitive control of an industrial biaxial precision gantry for contouring tasks, IEEE Transactions on Control Systems Technology 19(6): 1559-1568.

Inoue, T., Nakano, M. and Iwai, S. (1981). High accuracy control of a proton synchrotron magnet power supply, Proceedings of the 8th IFAC World Congress, Kyoto, Japan, pp. 216-221.

Khargonek, P.P., Petersen, I.R. and Zhou, K. (1990). Robust stabilization of uncertain linear systems: Quadratic stability and $\mathrm{H}_{\infty}$ control theory, IEEE Transactions on $\mathrm{Au}$ tomatic Control 35(37): 356-361.

Liu, J.Q., Chen, W.J., Wu, M., She, J. and He, Y. (2014a). Compensation in repetitive control system for aperiodic disturbances and input dead zone, 19th World Congress of the International Federation of Automatic Control, Cape Town, South Africa, pp. 2752-2757.

Liu, R.J., Liu, G.P., Wu, M., She, J. and Nie, Z.Y. (2014b) Robust disturbance rejection in modified repetitive control system, Systems and Control Letters 70: 100-108.

Miyazaki, T., Ohishi, K., Shibutani, I., Koide, D. and Tokumaru, H. (2006). Robust tracking control of optical disk recording system based on sudden disturbance observer, 32nd Annual Conference of the IEEE Industrial Electronics Society, Paris, France, pp. 5215-5220.

Pipeleers, G., Demeulenaere, B., Schutter, J.D. and Swevers, J. (2009). Generalised repetitive control: Relaxing the period-delay-based structure, IET Control Theory and Applications 3(11): 1528-1536.

She, J., Fang, M. and Ohyama, Y. (2008). Improving disturbance-rejection performance based on an equivalent-input-disturbance approach, IEEE Transactions on Industrial Electronics 55(1): 380-389.

She, J., Wu, M., Lan, Y.H. and He, Y. (2010). Simultaneous optimisation of the low-pass filter and state-feedback controller in a robust repetitive-control system, IET Control Theory and Applications 4(8): 1366-1376.

She, J., Xin, X. and Pan, P. (2011). Equivalent-input-disturbance approach-analysis and application to disturbance rejection in dual-stage feed drive control system, IEEE Transactions on Mechatronics 16(2): 330-340.

She, J., Zhou, L. and Wu, M. (2012). Design of a modified repetitive-control system based on a continuous-discrete 2D model, Automatica 48(5): 844-850.

Wu, M., Xu, B.G., Cao, W.H. and She, J. (2014). Aperiodic disturbance rejection in repetitive-control systems, IEEE Transactions on Control Systems Technology 22(3): 1044-1051. 
Yamamoto, Y. (1994). A function space approach to sampled data control systems and tracking problems, IEEE Transactions on Automatic Control 39(4): 703-713.

Zhou, K., Doyle, J.C. and Glover, K. (1996). Robust and Optimal Control, Prentice Hall, Inc., Upper Saddle River, NJ.

Zhou, L., She, J. and Wu, M. (2012). Design of a robust modified repetitive-control system for a periodic plant, ASME Journal of Dynamic Systems, Measurement, and Control 134(1): 011023-1-7.

Zhou, L., She, J., Wu, M. and He, Y. (2013). Design of a robust observer-based modified repetitive-control system, ASME Journal of Dynamic Systems, Measurement, and Control 52(3): 375-382.

Zhou, L., She, J., Wu, M., He, Y. and Zhou, S.W. (2014a). Estimation and rejection of aperiodic disturbance in a modified repetitive-control system, IET Control Theory and Applications 8(10): 882-889.

Zhou, L., She, J. and Zhou, S. (2014b). A 2D system approach to the design of a robust modified repetitive-control system with dynamic output-feedback controller, International Journal of Applied Mathematics and Computer Science 24(2): 325-334, DOI: 10.2478/amcs-2014-0024.

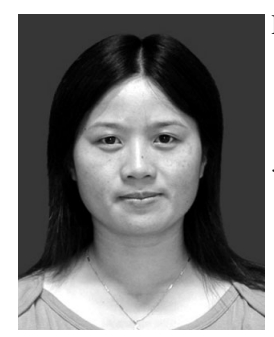

Lan Zhou received a B.Sc. degree in 1998 from Hunan Normal University, Changsha, China, and an M.Sc. degree in 2006 from Central South University, Changsha. From 2008 to 2010, she was a joint cultivation doctoral candidate of Japan and China. She received her Ph.D. degree in control science and engineering from Central South University in 2011. She is a professor of control theory and control engineering with the School of Information and Electrical Engineering, Hunan University of Science and Technology, Xiangtan, China. Her current research interests include robust control, repetitive control, and control application.

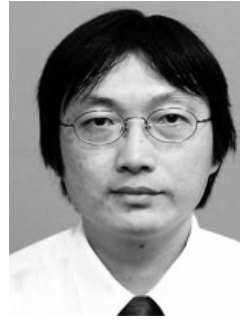

Jinhua She received a B.Sc. (1983) in engineering from Central South University, Changsha, Hunan, China, an M.S. (1990) and a Ph.D. (1993) in engineering from the Tokyo Institute of Technology, Japan. In 1993, he joined the School of Engineering, Tokyo University of Technology, where he is currently a professor. His research interests include application of control theory, repetitive control, process control, Internet-based engineering education, and robotics.

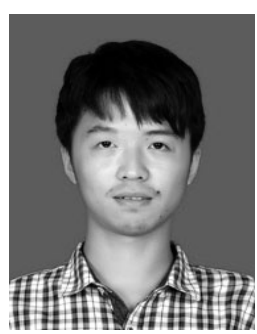

Chaoyi Li received a B.Sc. degree in 2014 from the Hunan University of Science and Technology, Xiangtan, China. Now he is studying for a Master's degree at the same university. His research interests include repetitive control, nonlinear system control, and the application of control theory.

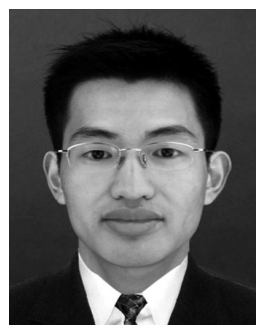

Changzhong Pan received a B.Sc. (2006) in engineering from Nanchang University, China, an M.Sc. (2009) and a Ph.D. (2013) in control theory and engineering from Central South University, Changsha, China. Since 2013, he has been with the School of Information and Electrical Engineering, Hunan University of Science and Technology, Xiangtan, China. From January 2011 to January 2013, he was a visiting scholar with the Advanced Robotics and Intelligent Systems Laboratory, University of Guelph, Canada. His research interests include nonlinear systems, robotics and robust control.

Received: 7 July 2015

Revised: 9 December 2015

Accepted: 26 January 2016 